\title{
A Generalized Recurrent Neural Network for Matrix Inversion
}

\author{
Jun Wang \\ Department of Industrial Technology \\ University of North Dakota \\ Grand Forks, ND 58202-7118, USA
}

Matrix inversion deals with computing the inverse matrix $A^{-1}$ of a given $n \times n$ nonsingular matrix $A$, which has been widely used in a variety of applications such as control, robotics, and signal processing. This paper presents a generalized recurrent neural network for matrix inversion. The generalized recurrent neural network can use any monotone nondecreasing activation functions and its steady states are guaranteed to present the inverse matrix $A^{-1}$.

The dynamics of the recurrent neural network for matrix inversion can be described by the following matrix-valued differential and algebraic equations,

$$
\begin{aligned}
\frac{d V(t)}{d t} & =-\mu A^{T} U(t), \\
U(t) & =G[A V(t)-I],
\end{aligned}
$$

where $A^{T}$ denotes the transpose of $A, V(t)$ and $U(t)$ are $n \times n$ activation state matrices of the neurons in the output and input layers respectively, $\mu>0$ is a scalar gain parameter, $G[X]=\left[g_{i j}\left(x_{i j}\right)\right]$ is a matrix of activation functions, and $I$ is the identity matrix.

The architecture of the recurrent neural network consists of two layers and each layer consists of an $n \times n$ array of neurons. Each output neuron represents an element in the inverse matrix $A^{-1}$. The connection weight matrix from the input layer to the output layer is defined as $-\mu A^{T}$ and the connection weight matrix from the output layer to the input layer is defined as $A$. The biasing threshold (constant input) matrix in the input layer is defined as $I$ and there are no biases for the neurons in the output layer. There is a functional transformation for each neuron in the input layer and an integral transformation for each neuron in the output layer.

The dynamical equations show that $v_{i j}(t)$ is connected with $u_{1 j}(t), u_{2 j}(t), \ldots, u_{n j}(t)$ only and $u_{i j}(t)$ is connected with $v_{1 j}(t), v_{2 j}(t), \ldots, v_{n j}(t)$ only. This pattern of connectivity shows the proposed recurrent neural network can actually be decomposed into $n$ independent subnetworks. Each subnetwork represents one column vector of $A^{-1}$. The dynamical equations also indicate that the connection weight matrices are identical for each subnetwork.

Main Result: If $g_{i j}\left(x_{i j}\right)$ is monotone nondecreasing with respect to $x_{i j}$ (i.e., $d g_{i j} / d x_{i j} \geq 0$ ) and $g_{i j}\left(x_{i j}\right)=0$ is equivalent to $x_{i j}=0$ (i.e., $g_{i j}\left(x_{i j}\right)=0$ iff $x_{i j}=0$ ) for $i, j=1,2 \ldots, n$, then the recurrent neural network for matrix inversion is asymptotically stable in the large and its equilibrium states represent the inverse matrix of a given nonsingular matrix (i.e., $\forall V(0) \in R^{n \times n}, \lim _{t \rightarrow \infty} V(t)=A^{-1}$ ).

The analytical result has been substantiated by simulation results. 